\title{
OVARIOHISTERCTOMIA EM LEITOA (Sus scrofa domesticus)
}

\author{
Caroline Marques da Silva ${ }^{1}$ \\ Racquel Andrade Fernandes ${ }^{1}$ \\ Caio Cavalcanti Balençuela ${ }^{1}$ \\ Fabio Futema ${ }^{2}$ \\ Daniel de Souza Ramos Angrimani ${ }^{2}$ \\ Milena Rodrigues Soares ${ }^{2}$ \\ Simone Rodrigues Ambrosio ${ }^{2}$ \\ Marcio Augusto Ferreira ${ }^{2}$
}

\begin{abstract}
RESUMO
A espécie suína nos últimos anos vem sendo espécie de escolha como companhia doméstica. $\mathrm{Na}$ Medicina Veterinária conhecemos o crescimento da suinocultura e as melhorias necessárias para o aumento da exportação. Melhorias na alimentação, melhoramento genético, no manejo e procedimentos cirúrgicos em machos. Pensando na domesticação desses animais e manutenção da saúde dessa espécie animal, além do manejo alimentar, se faz necessário novos estudos para a melhor adaptação desses animais e também o controle da reprodução em ambientes domésticos sem o interesse econômico da espécie. O procedimento cirúrgico para o controle da reprodução de fêmeas é a retirada dos ovários (ovariectomia) e poucos estudos foram descritos, tanto sobre o procedimento, quanto estudos comparativos para o melhor procedimento cirúrgico. A Ovariossalpingohisterectomia é um dos procedimentos cirúrgicos eletivos mais realizados na rotina veterinária de pequenos animais, sendo um meio eficaz no controle populacional e de prevenção de doenças. Devido à ausência de descrição da técnica cirúrgica em animais de produção, o presente trabalho relata o procedimento de ovariossalpingohisterectomia realizado em leitoa (Sus scrofa domesticus) de aproximadamente quatro meses de idade, submetida a anestesia geral, com a avaliação pressórica e a hemogasometria, garantindo indicadores de bem estar animal e controle dos parâmetros vitais durante todo o procedimento, podendo ser alternativa para abordagens cirúrgicas de animais da espécie suína de companhia doméstica, conhecidos como minipig (minisuíno).
\end{abstract}

Palavras-Chave: Esterilização Reprodutiva; Técnica; Cirurgia; Mini-pig;

\section{OVARIOHYSTERECTOMY IN PIGLE (Sus scrofa domesticus)}

\begin{abstract}
The swine species in the last years has been the species of choice as a domestic companion. In Veterinary Medicine we know the growth of the swine industry and the improvements needed to increase exportation. Improvements in feeding, genetic improvement, management, and surgical procedures in males. Thinking about the domestication of these animals and the maintenance of the health of this animal species, besides the food management, new studies are necessary for a better adaptation of these animals and also the control of reproduction in domestic environments without the economic interest of the species. The surgical procedure for the control of female reproduction is the removal of the ovaries (ovariectomy) and few studies have been described, both on the procedure and comparative studies for the best

${ }^{1}$ Graduando em Universidade São Judas *Correspondência. marquescaroline34@gmail.com

${ }^{2}$ Docente Universidade São Judas. fabiofutema@uol.com.br

Silva CM, Fernandes RA, Balençuela CC, Futema F, Angremani DSR, Soares MR, et al. Ovariohisterctomia em leitoa (Sus scrofa domesticus) Vet. e Zootec. 2021; v28: 001-009.
\end{abstract}


surgical procedure. Ovariosalpingohysterectomy is one of the most performed elective surgical procedures in small animal veterinary routine, being an effective means of population control and disease prevention. Due to the absence of description of the surgical technique in production animals, the present work reports the procedure of ovariosalpingohysterectomy performed in a female pig (Sus scrofa domesticus) of approximately four months of age, submitted to general anesthesia, with pressure evaluation and hemogasometry, ensuring indicators of animal welfare and control of vital parameters during the entire procedure, which may be an alternative for surgical approaches to animals of the domestic swine species, known as minipigs.

Keywords: Reproductive Sterilization; Technique; Veterinary Surgery; Mini-pig;

\section{OVARIOHISTERCTOMÍA EN CERDO (Sus scrofa domesticus)}

\section{RESUMEN}

En los últimos años, la especie porcina ha sido la elegida como compañero doméstico. En Veterinaria conocemos el crecimiento de la ganadería porcina y las mejoras necesarias para aumentar las exportaciones. Mejoras en la alimentación, mejora genética, gestión y procedimientos quirúrgicos en los machos. Pensando en la domesticación de estos animales y en el mantenimiento de la salud de esta especie animal, además del manejo de la alimentación, son necesarios nuevos estudios para una mejor adaptación de estos animales y también el control de la reproducción en ambientes domésticos sin el interés económico de la especie. El procedimiento quirúrgico para el control de la reproducción de las hembras es la extirpación de los ovarios (ovariectomía) y se han descrito pocos estudios, tanto sobre el procedimiento como estudios comparativos para el mejor procedimiento quirúrgico. La ovariosalpingohisterectomía es uno de los procedimientos quirúrgicos electivos más realizados en la rutina veterinaria de los pequeños animales, siendo un medio eficaz de control poblacional y de prevención de enfermedades. Debido a la ausencia de descripción de la técnica quirúrgica en animales de producción, el presente trabajo reporta el procedimiento de ovariosalpingohisterectomía realizado en un lechón (Sus scrofa domesticus) de aproximadamente cuatro meses de edad, sometido a anestesia general, con evaluación de la presión y hemogasometría, garantizando los indicadores de bienestar animal y el control de los parámetros vitales durante todo el procedimiento, lo que puede ser una alternativa para el abordaje quirúrgico de animales de la especie porcina doméstica, conocida como minipig (minicerdo).

Palabras Clave: Esterilización Reproductiva; Técnica; Cirurgía; Minicerdo;

\section{INTRODUÇÃO}

Os suínos ganham destaque em todo o mundo dentro da agropecuária, onde, na suinocultura há o maior enfoque para reprodução e consumo destes animais, tendo em vista que a carne suína é a mais consumida em todo o mundo, e o Brasil vem se consolidando como um dos maiores produtores globais dessa proteína ${ }^{1}$. Todavia, este cenário vem sendo modificado com a domesticação da espécie (1), principalmente com a raça mini-pig, onde estes animais estão sendo inseridos como pets e assessorados na rotina clínica de pequenos animais.

A ovariohisterectomia $(\mathrm{OH})$ destaca-se dentre os procedimentos cirúrgicos mais realizados na prática veterinária de animais de companhia, em cadelas e gatas (2). Tal técnica 
visa a remoção dos ovários, tubas uterinas e útero, resultando na esterilização das fêmeas (2). A principal abordagem realizada em pequenos animais, é pela linha mediana ventral, consistindo em uma celiotomia ventral da linha média, no terço médio entre o púbis e o umbigo. Os ovários são exteriorizados, e procede-se a ligadura dos pedículos ovarianos e uterino com auxílio de pinças hemostáticas e material de sutura absorvível, podendo ser empregadas a ligadura simples, dupla e tripla, seguida de incisões aproximadas às ligaduras e retirada dos órgãos (2).

Metodologias mais atuais são empregadas para a esterilização de cadelas, como descreve o estudo realizado por Sánchez-Margallo et al. (2015), os quais abordaram uma técnica de ovariohisterectomia realizada por meio de laparoscopia, com incisão única mediana pré-umbilical, a qual permite a utilização de quatro instrumentos através de um único canal. Pontua-se neste estudo, uma manipulação reduzida de vísceras intestinais, com consequente redução da dor pós-operatória, menor tempo cirúrgico, com melhor recuperação e reduzido tempo de hospitalização; devido à excelente observação dos órgãos em sua topografia anatômica, facilitando a execução das ligaduras e retirada dos órgãos.

Entretanto, em suínos a técnica de ovariectomia (OVE) é a mais utilizada, onde ocorre a retirada cirúrgica dos ovários por meio de uma incisão lateral na região do flanco paralombar, sendo que, a principal indicação é cessar a capacidade reprodutiva da fêmea (2). Salienta-se que esta técnica é menos invasiva e demanda menor tempo de recuperação para o animal, se comparada à $\mathrm{OH}$, além do custo-benefício se tratando dos criadores. A desvantagem da OVE em relação a $\mathrm{OH}$ é de não prevenir o desenvolvimento de tumores uterinos (3), como vantagem, permite a observação da ferida cirúrgica à distância, muito útil no monitoramento do pós-operatório de animais ariscos, e reduz a evisceração dos órgãos abdominais nos casos de deiscência de sutura (3). Ainda, a ovariohisterectomia também é eficiente quando se trata na prevenção do nascimento de ninhadas indesejadas, fator importante para o proprietário e controle populacional da espécie (3). Contudo, maiores descrições da técnica ainda perfazem necessárias (2).

Técnica similar foi executada em um experimento feito por Quessada et al. (2009), a qual denominada como técnica experimental de ovariohisterectomia em cadelas, foram realizadas três incisões, duas paramedianas (direita e esquerda) para ligadura dos ovários e uma retroumbilical, para ligadura do útero e retirada dos órgãos. Avaliou-se o cortisol sérico das fêmeas submetidas ao experimento, e pôde-se concluir que, comparada à técnica tradicional de ovariohisterectomia em fêmeas, a técnica experimental desencadeia um maior estresse devido à grande manipulação dos órgãos, sendo indicada para campanhas de esterilização com alta demanda.

Portanto, o objetivo do presente relato foi descrever a técnica de ovariohisterectomia em uma fêmea da espécie Sus scrofa domesticus, a fim de instruir os cirurgiões na abordagem da mesma, principalmente em uma aplicação na raça Mini-Pig. Ademais, este trabalho descreve, também, a eficiência da associação de fármacos para promover anestesia e analgesia satisfatórias no animal, visando o bem-estar do mesmo.

\section{MATERIAIS E MÉTODOS}

Avaliação pré-operatória de esterilização eletiva de uma leitoa da raça White Large foi realizada no setor de Clínica Médica e Cirúrgica de Grandes Animais do Hospital Veterinário da Universidade São Judas Tadeu, o animal tinha, aproximadamente, quatro meses de idade e pesava $25 \mathrm{~kg}$. Durante a anamnese a tutora relatou que o animal era domesticado e criado em grupo misto (machos e fêmeas) sem a intenção de reprodução, optando assim pela castração da mesma. No exame clínico não foram detectadas irregularidades quanto ao quadro físico do animal. A paciente encontrava-se saudável, hidratada e sem sinais de estro. 
Neste cenário optou-se pela realização da técnica cirúrgica de ovariohisterectomia. Para tal, a paciente foi mantida em jejum hídrico de quatro horas e alimentar de oito horas. $\mathrm{O}$ protocolo anestésico instituído deu-se início com base na aplicação de Midazolam $(0,6$ $\mathrm{mg} / \mathrm{kg}$ ), Quetamina (2 mg/kg) e Acepromazina $(0,1 \mathrm{mg} / \mathrm{kg}$ ) via intramuscular (4). O efeito sedativo dos medicamentos foi eficaz, prosseguindo com a tricotomia ampla da área a ser acessada e a colocação do acesso venoso em veia marginal do conduto auditivo com cateter $20 \mathrm{G}$ e fluidoterapia com ringer lactato na velocidade de $5 \mathrm{ml} / \mathrm{kg} / \mathrm{hora}$.

Em seguida, foi administrado Propofol $(3,2 \mathrm{mg} / \mathrm{kg})$ intravenoso, promovendo a indução anestésica (4). A ação do fármaco deu-se em dois minutos, possibilitando a intubação orotraqueal com sonda número 7,5 através do fibroscópio. A manutenção anestésica seguiu por meio do uso de Isoflurano diluído em $\mathrm{O}_{2}$ a $100 \%$, em circuito sem reinalação. A paciente manteve-se em plano anestésico durante todo o procedimento. Após instalação dos equipamentos de monitorização, o animal foi posicionado em decúbito lateral direito para realização da técnica de bloqueio do músculo quadrado lombar, onde foram realizadas a tricotomia e antissepsia das áreas da região lateral (5). A técnica ocorreu com o auxílio de ultrassom acoplado a transdutor linear com frequência intermediária (5-12 MHz), posicionado entre as vértebras L2 e L3. Em seguida, foi inserida uma agulha de Tuohy $22 \mathrm{G}$ "in plane", sendo determinada a borda dorso-ventral como ponto de introdução (Figura 1). O local de inserção foi identificado entre o músculo quadrado lombar e o músculo psoas maior, e administrados $20 \mathrm{ml}$ de Bupivacaína (0,5\%) bilateralmente (6).

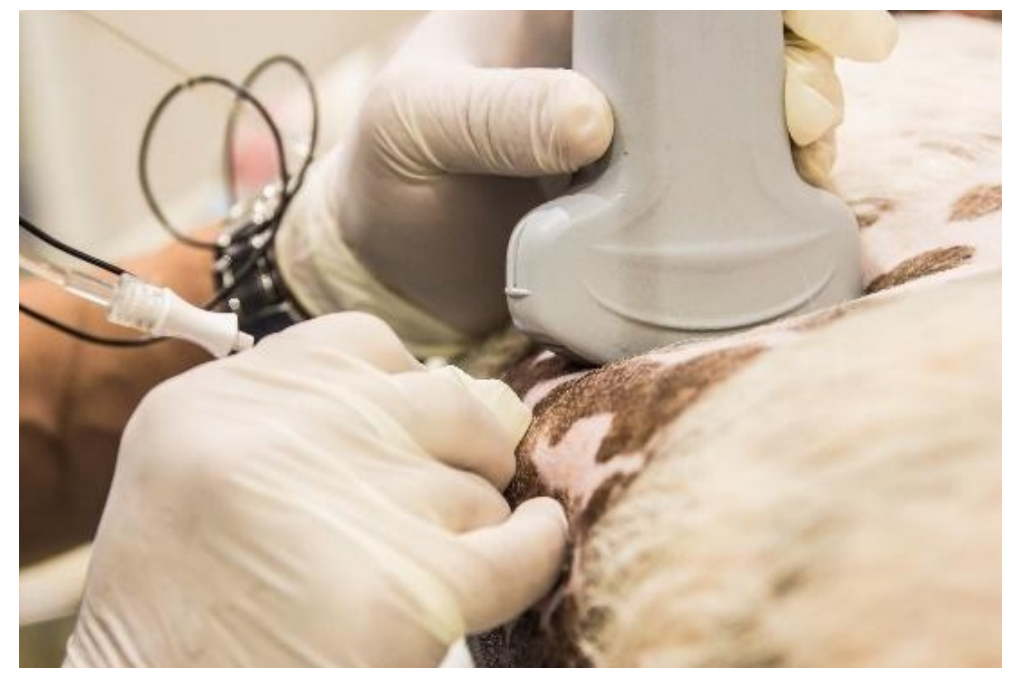

Figura 1. Técnica de bloqueio do músculo quadrado lombar. Introdução da agulha Tuohy, guiada por ultrassom, entre as vértebras L2 e L3.

No período trans-operatório, foram realizadas avaliações dos parâmetros vitais, como a frequência cardíaca e respiratória, capnografia, pressões arteriais sistólica, diastólica e média e saturação de oxigênio, os quais se mantiveram dentro do valor de referência para a espécie (7). Concomitante com tais observações, foi realizada a avaliação hemogasométrica da paciente, onde foram coletadas três amostras de sangue arterial, com intervalo de uma hora entre cada uma, cuja as duas primeiras foram puncionadas com a paciente em plano anestésico, entubada e com oferta de $100 \%$ de oxigênio e a terceira coleta com o animal já em recuperação anestésica (Tabela 1). As amostras foram processadas e analisadas no Equipamento Analisador de Gases Sanguíneos, modelo ABL 90 da marca Radiometer Medical ApS. Para a análise hematológica (Tabela 2) e bioquímica (Tabela 3) foram realizadas quatro coletas de amostras sanguíneas no período pré-cirúrgico, no trans-cirúrgico, ao término do procedimento e, por fim, a última coleta para realização do exame hemograma, trinta minutos após o término da cirurgia. 
Para a realização do procedimento cirúrgico, o animal foi posicionado em decúbito dorsal, com posterior antissepsia de toda área abdominal realizada com clorexidina e álcool $70 \%$. A técnica foi realizada como a descrita em cadelas por Fossum (2).

Tabela 1. Valores obtidos através da mensuração hemogasométrica das amostras sanguíneas arteriais.

\begin{tabular}{|c|c|c|c|}
\hline & Primeira Coleta & Segunda Coleta & Terceira Coleta \\
\hline $\mathbf{p C O}_{2}$ & $40,5 \mathrm{mmHg}$ & $46,9 \mathrm{mmHg}$ & $39,8 \mathrm{mmHg}$ \\
\hline $\mathbf{p \mathbf { O } _ { 2 }}$ & $440 \mathrm{mmHg}$ & $340 \mathrm{mmHg}$ & $145 \mathrm{mmHg}$ \\
\hline $\mathbf{p H}$ & 7,47 & 7,45 & 7,53 \\
\hline $\mathbf{N a}$ & $131 \mathrm{mmol} / \mathrm{L}$ & $129 \mathrm{mmol} / \mathrm{L}$ & $128 \mathrm{mmol} / \mathrm{L}$ \\
\hline $\mathbf{K}$ & $2,68 \mathrm{mmol} / \mathrm{L}$ & $2,60 \mathrm{mmol} / \mathrm{L}$ & $2,62 \mathrm{mmol} / \mathrm{L}$ \\
\hline $\mathbf{C a}$ & $5,06 \mathrm{mg} / \mathrm{dL}$ & $5,10 \mathrm{mg} / \mathrm{dL}$ & $4,90 \mathrm{mg} / \mathrm{dL}$ \\
\hline $\mathbf{C l}$ & $93 \mathrm{meq} / \mathrm{L}$ & $88 \mathrm{meq} / \mathrm{L}$ & $88 \mathrm{meq} / \mathrm{L}$ \\
\hline
\end{tabular}

Tabela 2. Valores obtidos através da realização do exame hemograma de amostras sanguíneas venosas.

\begin{tabular}{|c|c|c|c|c|c|}
\hline Exame & $\begin{array}{c}\text { Resultados } \\
\text { Pré-cirúrgico }\end{array}$ & $\begin{array}{c}\text { Resultados } \\
\text { Trans cirúrgico }\end{array}$ & $\begin{array}{c}\text { Resultados } \\
\text { Pós- cirúrgico }\end{array}$ & $\begin{array}{c}\text { Resultados } \\
\text { após 30 } \\
\text { minutos }\end{array}$ & $\begin{array}{c}\text { Valores de } \\
\text { Referência }\end{array}$ \\
\hline Eritrócitos & 5,73 & 4,77 & 4,77 & 4,49 & 5,0 a 8,0 \\
\hline Hemoglobina & 8,6 & 7,1 & 7,2 & 6,79 & 10,0 a 16,0 \\
\hline Hematócrito & 28,6 & 23,7 & 23,7 & 21,9 & 32,0 a 50,0 \\
\hline VCM & 49,8 & 49,7 & 49,8 & 48,9 & 50 a 68 \\
\hline HCM & 15,0 & 14,8 & 15,0 & 14,9 & 17,4 \\
\hline CHCM & 30,1 & 29,9 & 30,3 & 30,5 & 30,0 a 34,0 \\
\hline Leucócitos & 12.500 & 13.700 & 8.100 & 7.500 & 11.000 a 22.000 \\
\hline Segmentados & 3.625 & 5.480 & 3.240 & 3.300 & 3.200 a 10.000 \\
\hline Eosinófilos & 250 & 0 & 0 & 0 & 50 a 2.000 \\
\hline Linfócitos & 375 & 959 & 0 & 250 & 4.500 a 13.000 \\
\hline Monócitos & 8.250 & 7.261 & 4.860 & 3.975 & 250 a 2.500 \\
\hline Plaquetas & 303.000 & 336.000 & 294.000 & 280.000 & \\
\hline
\end{tabular}

Tabela 3. Valores obtidos através da realização do exame bioquímica de amostras sanguíneas venosas.

\begin{tabular}{|c|c|c|c|c|c|}
\hline Exame & $\begin{array}{c}\text { Resultados } \\
\text { Pré-cirúrgico }\end{array}$ & $\begin{array}{c}\text { Resultados } \\
\text { Trans cirúrgico }\end{array}$ & $\begin{array}{c}\text { Resultados } \\
\text { Pós-cirúrgico }\end{array}$ & $\begin{array}{c}\text { Resultados } \\
\text { após 30 } \\
\text { minutos }\end{array}$ & $\begin{array}{c}\text { Valores de } \\
\text { Referência }\end{array}$ \\
\hline Albumina & $1,94 \mathrm{~g} / \mathrm{dL}$ & $1,29 \mathrm{~g} / \mathrm{dL}$ & $1,29 \mathrm{~g} / \mathrm{dL}$ & Não realizado & $1,80 \mathrm{a} 3,30 \mathrm{~g} / \mathrm{dL}$ \\
\hline Globulina & $0,43 \mathrm{~g} / \mathrm{dL}$ & $0,26 \mathrm{~g} / \mathrm{dL}$ & $0,18 \mathrm{~g} / \mathrm{dL}$ & Não realizado & $5,29 \mathrm{a} 6,43 \mathrm{~g} / \mathrm{dL}$ \\
\hline $\begin{array}{c}\text { Proteínas } \\
\text { Totais }\end{array}$ & $2,37 \mathrm{~g} / \mathrm{dL}$ & $1,55 \mathrm{~g} / \mathrm{dL}$ & $1,47 \mathrm{~g} / \mathrm{dL}$ & Não realizado & 7,90 a $8,90 \mathrm{~g} / \mathrm{dL}$ \\
\hline Glicose & $139 \mathrm{mg} / \mathrm{dL}$ & $137 \mathrm{mg} / \mathrm{dL}$ & $230 \mathrm{mg} / \mathrm{dL}$ & $159 \mathrm{mg} / \mathrm{dL}$ & 85 a $150 \mathrm{mg} / \mathrm{dL}$ \\
\hline ALT & $110 \mathrm{U} / \mathrm{L}$ & $86 \mathrm{U} / \mathrm{L}$ & $93 \mathrm{U} / \mathrm{L}$ & $64 \mathrm{U} / \mathrm{L}$ & 31 a $58 \mathrm{U} / \mathrm{L}$ \\
\hline AST & $66 \mathrm{U} / \mathrm{L}$ & $60 \mathrm{U} / \mathrm{L}$ & $62 \mathrm{U} / \mathrm{L}$ & $52 \mathrm{U} / \mathrm{L}$ & 32 a $84 \mathrm{U} / \mathrm{L}$ \\
\hline $\begin{array}{c}\text { Fosfatase } \\
\text { Alcalina }\end{array}$ & $549 \mathrm{U} / \mathrm{L}$ & $482 \mathrm{U} / \mathrm{L}$ & $596 \mathrm{U} / \mathrm{L}$ & $375 \mathrm{U} / \mathrm{L}$ & 118 a $395 \mathrm{U} / \mathrm{L}$ \\
\hline \begin{tabular}{c} 
Colesterol \\
\hline
\end{tabular} & $53 \mathrm{mg} / \mathrm{dL}$ & $67 \mathrm{mg} / \mathrm{dL}$ & $60 \mathrm{mg} / \mathrm{dL}$ & $28 \mathrm{mg} / \mathrm{dL}$ & $36 \mathrm{a} 54 \mathrm{mg} / \mathrm{dL}$ \\
\hline
\end{tabular}

Após o posicionamento dos panos de campo deu-se início à cirurgia. A incisão de pele foi realizada na linha média por meio de incisão retro-umbilical de aproximadamente cinco centímetros (Figura 2A). Em seguida realizou-se a divulsão do tecido subcutâneo e adiposo (Figura 2B). O acesso a cavidade abdominal se deu por meio de uma incisão invertida na linha alba e ampliada com o auxílio de tesoura. O omento foi elevado e tracionado para 
facilitar a identificação das estruturas. Devido ao acúmulo de conteúdo nas alças intestinais, dificultando a observação dos ovários e do útero, optou-se por prolongar a incisão realizada anteriormente. Com o auxílio de gancho com esfera foi possível identificar os cornos uterinos (Figura 2C) e posteriormente o ovário direito, localizado caudalmente ao rim direito, onde este foi isolado com gaze cirúrgica e procedeu-se com a ligadura do complexo arteriovenoso ovariano (CAVO) com fio nylon 3-0 (Figura 2D).

Após a ligadura foi seccionado o pedículo ovariano, onde o mesmo foi inspecionado em busca de possíveis hemorragias. Constatada a ausência, foi seccionado o excesso do fio cirúrgico e o pedículo foi reposicionado na cavidade abdominal. $\mathrm{O}$ mesmo procedimento foi repetido para a retirada do ovário esquerdo. Após a secção dos dois ovários, o corpo uterino foi exposto, havendo a necessidade da ruptura do ligamento largo do útero, a fim de facilitar a sua manipulação. Foi realizada a ligadura do corpo do útero cranialmente à cérvix (Figura 2E), o que permitiu a completa remoção do endométrio. A ligadura no útero foi realizada com fio nylon 2-0, por transfixação, incluindo também a veia e artéria uterina. Posteriormente o coto do útero foi seccionado e inspecionado, onde não foi observada a presença de hemorragia (Figura 2F). A musculatura abdominal foi suturada com fio nylon 2-0 em padrão simples interrompido (Figura 2G) e a pele com fio nylon 2-0 em padrão Wolf interrompido (Figura $2 \mathrm{H})$. Ao final da cirurgia foram administrados Meloxicam $(0,1 \mathrm{mg} / \mathrm{kg})$ e Dipirona $(25 \mathrm{mg} / \mathrm{kg})$ por via endovenosa, e Penicilina (40.000 UI/kg) por via intramuscular.

Após sete dias da realização da cirurgia, os pontos da pele foram retirados e observou-se a completa cicatrização da ferida cirúrgica do animal.

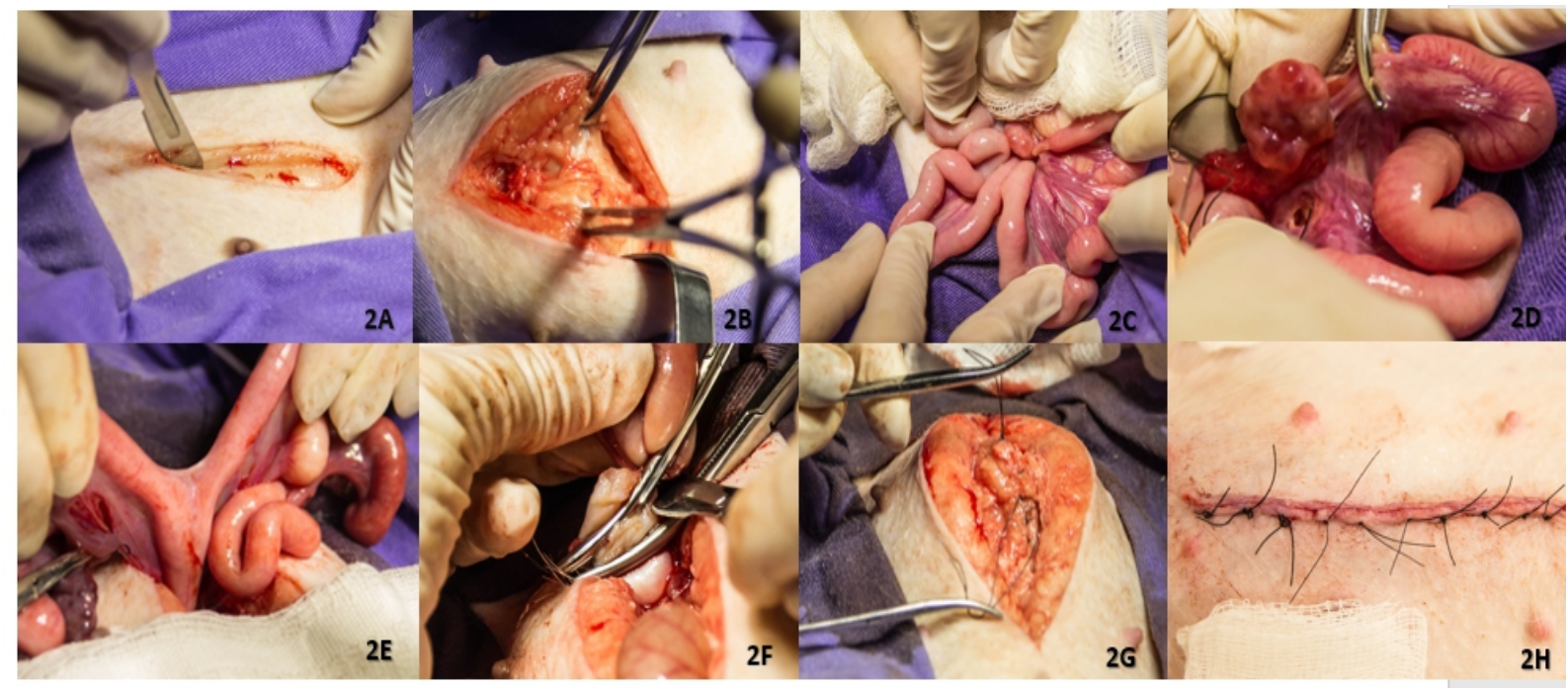

Figura 2. Ovariohisterectomia em leitoa (Sus domesticus). A) Incisão retro-umbilical em linha média. B) Divulsão do tecido subcutâneo e adiposo. C) Exposição dos cornos uterinos. D) Ligadura do complexo arteriovenoso ovariano (CAVO). E) Ligadura do corpo do útero. F) Secção do coto do útero. G) Sutura de musculatura em padrão simples interrompido. H) Sutura de pele em padrão Wolf interrompido.

\section{DISCUSSÃO}

Ao avaliar os dados mensurados nos exames hemogasométricos, é possível constatar que os gases sanguíneos se mantiveram em valores consideravelmente normais para a espécie, segundo Gianotti et al. (8), tendo em vista que a paciente se encontrava em plano anestésico, com oferta de $100 \%$ de oxigênio nas primeiras coletas. Além disso, é válido ressaltar a redução no valor de $\mathrm{pO}_{2}$ na última amostra, devido a paciente já se encontrar em recuperação anestésica, desvinculada do aparelho de oxigênio (8). Em contrapartida, os valores dos 
eletrólitos avaliados se apresentaram abaixo do parâmetro referencial e os índices obtidos através da mensuração do $\mathrm{pH}$ sanguíneo se mostraram estáveis. Considerando o protocolo alimentar que era instituído para o animal, determinado por uma dieta vegana, pressupõe-se que a deficiência de eletrólitos apresentada no exame hemogasométrico, seja proveniente de uma alimentação desbalanceada. A dieta era composta por legumes, vegetais e cereais, e de acordo com Merchy (9), o equilíbrio eletrolítico dos fluidos sanguíneos depende de diversos fatores, como nível proteico alimentar, suplementos de $\mathrm{Na}+$ e alterações na composição da alimentação quanto ao balanço eletrolítico.

O hemograma consiste na avaliação das hemácias, leucócitos e plaquetas, onde muitas vezes é associado ao exame de bioquímica sanguínea para marcadores hepáticos com intuito de avaliar alterações no fígado, através das enzimas ALT e AST que estão presentes no citoplasma dos hepatócitos e músculos (10). A fosfatase alcalina é sintetizada nos hepatócitos e osteoblastos, a glicose é produzida no pâncreas, porém uma parte é sintetizada no fígado através de gliconeogênese e as proteínas totais, como a albumina e globulina, são produzidas pelo fígado (11). O eritrograma, leucócitos totais e plaquetograma foram realizados pela máquina automatizada BC-2800Vet da marca Mindray e a contagem diferencial de leucócitos através de extensão sanguínea e microscopia óptica comum para avaliação da morfologia celular e coloração. A análise da bioquímica sanguínea de fator hepático foi mensurada através da máquina automatizada LABMAX 100 da marca Labtest. Com o resultado do hemograma podemos perceber algumas alterações de acordo com os valores de referência da pesquisa realizada por Prado (11), onde se constatou que o eritrograma, hemoglobina, eritrócitos, HCM e VCM estavam abaixo do valor esperado, porém, como já mencionado, o animal era submetido à uma dieta restrita, consequentemente, níveis protéicos poderiam sofrer alterações, e há necessidade de uma investigação clínica e laboratorial para poder correlacionar essas alterações hematológicas com os componentes da alimentação, necessitando assim, maiores pesquisas na área para evidenciação científica dos dados.

$\mathrm{O}$ uso da técnica de ovariohisterectomia nesse animal se fez necessário devido à escassez de descrições para a espécie (Sus scrofa domesticus). Tal procedimento foi escolhido com o intuito de esterilizar a paciente, reduzindo, assim, os riscos de doenças reprodutivas (2).

Todavia, ao realizar o procedimento cirúrgico deve-se considerar as diferenças anatômicas dos suínos, sendo necessário o conhecimento da topografia das estruturas presentes na cavidade abdominal. $\mathrm{O}$ aparelho reprodutor da porca possui tubas uterinas que se apresentam como um longo par de tubos envelopados medindo entre 15 a 30 centímetros (13). Por sua vez, o útero é composto pelo corpo, cérvix e dois cornos, apresentando-se com cerca de 5 centímetros de comprimento cada um. A cérvix possui aproximadamente 10 centímetros de comprimento, sendo extremamente longa e flexuosa, disposta em numerosas espirais, assemelhando-se ao intestino delgado (13). O ligamento largo do útero é descrito como mesométrio, o qual é responsável pela sustentação do útero no peritônio, podendo ser facilmente confundido com o mesentério, devido a sua conformação anatômica e distribuição vascular (13). Já os ovários estão localizados em região caudal ao rim, sendo móveis e irregulares, com corpos lúteos e folículos projetados em sua superfície, dando-lhes aparência lobulada (13).

Para o procedimento de $\mathrm{OH}$, é ideal que o animal esteja em jejum de 12 a 16 horas de alimentos sólidos, pois devido ao metabolismo lento, a presença de conteúdo nas alças intestinais pode dificultar a manipulação e localização do trato reprodutivo durante o transoperatório (14). A incisão cirúrgica deve possuir de 7 a 10 centímetros para facilitar a abordagem abdominal (14). Ainda, o manuseio dos instrumentais para a abertura das camadas da pele se torna dificultoso, devido à friabilidade da espessa camada de tecido adiposo que esses animais possuem (14), tal como, observado nesse caso. 
As técnicas anestésicas em suínos têm sido pouco abordadas nos últimos anos. Torna-se provável que esse efeito esteja ligado à criação do tipo industrial, em que a espécie, por menor problema observado, estando passível para consumo, é encaminhado para o abate (6). No entanto, neste trabalho, tornou-se possível a determinação de uma combinação de fármacos anestésicos, os quais promoveram analgesia e dessensibilização adequada, além de agregar valor ao bem estar da paciente. A associação da técnica de bloqueio do músculo quadrado lombar, adaptada segundo Massone (5), tem como intuito estabelecer um controle da dor do paciente, possibilitando uma analgesia trans e pós-operatória. Ademais, esta técnica visa reduzir os volumes de fármacos anestésicos utilizados durante o procedimento cirúrgico e reduzir a necessidade de medicamentos opióides no pós-operatório (4). A técnica do bloqueio do músculo quadrado lombar foi aplicada pela primeira vez na espécie suína, neste estudo, sendo mais comum na rotina anestésica de cães e gatos.

\section{CONCLUSÃO}

O presente relato trouxe em questão a descrição da técnica de ovariohisterectomia em leitoa, até então não descrita na literatura. Tal técnica se mostrou de fácil execução e abordagem, desde que, haja período de jejum maior que doze horas. Ainda se observou a ausência de hemorragia e reação tecidual. Assim sendo, a $\mathrm{OH}$ apresentou-se como técnica válida para castração de fêmeas suínas.

\section{REFERÊNCIAS}

1. Girotto AF, Santos Filho JI. Embrapa suínos e aves. Concórdia: Embrapa Suínos e Aves; 2000 .

2. Fossum TW. Cirurgia de pequenos animais. 4a ed. Rio de Janeiro: Guanabara Koogan; 2014.

3. Quessada AM. Comparação de técnicas de ovariosalpingohisterectomia em cadelas. Acta Sci Vet. 2009;37:253-8.

4. Spinosa HS, Górniak SL, Bernadini MM. Farmacologia aplicada à Medicina Veterinária. 6a ed. Rio de Janeiro: Guanabara Koogan; 2017.

5. Massone F. Anestesiologia veterinária: farmacologia e técnicas: atlas e texto colorido. Rio de Janeiro: Guanabara Koogan; 2018.

6. Dhanjal S, Tonder S. Quadratus lumborum block. Theasure Island: StatPearls Publishing; 2019.

7. Sá M, Cardoso JM, Reis H, Esteves M, Sampaio J, Gouveia I, et al. Quadratus lumborum block: are we aware of its side effects? A report of 2 cases. Braz J Anesthesiol. 2018;68:396-9.

8. Gianotti GC, Beheregaray WK, Bianchi SP, Mombach V. Suíno como modelo experimental na pesquisa biomédica: valores fisiológicos normais. Acta Sci Vet. 2010;38:133-13. 
9. Weiss DJ, Wardrop KJ, editors. Schalm's veterinary hematology. 6th ed. Ames: WileyBlackwell; 2010.

10. Kaneko JJ. Clinical biochemistry of domestic animals. London: Academic; 1998.

11. Prado AMRB. Valores hematimétricos normais em suínos das raças landrace e largewhite no Paraná. Rev Acad Cienc Anim. 2002;2:65-80.

12. SánchezMargallo FM, Tapia-Araya A, Díaz-Güemes I. Preliminary application of a single-port access technique for laparoscopic ovariohysterectomy in dogs. Vet Rec Open 2015;2:e00153. doi:10.1136/ vetreco-2015-000153.

13. Spurgeon TL, Mccracker TO, Kainer RA. Atlas colorido de anatomia de grandes animais. Guanabara: Koogan; 2004.

14. Sousa LH, Sousa Filho LH, Sousa VM, Sousa JAG, Tamura S, Chalela D Jr. Endoscopic cholecystectomy: proposal of a new techinique totally natural orifices transluminal. Preliminary result of experimental study in pigs. Rev Bras Videocirurgia [Internet]. 2007 [citado 23 Out 2020];5:90-102. Disponível em: https://www.sobracil.org.br/revista/rv050502/artigo06.htm

15. Cunningham JG. Tratado de fisiologia veterinária. 3a ed. Rio de Janeiro: Guanabara Koogan; 2004. Equilíbrio ácido-básico.

16. Guyton AC. Tratado de fisiologia médica. 9a ed. Rio de Janeiro: Guanabara Koogan; 2002.

17. Sesti L, Sobestiansky J, Barcellos DESN. Suinocultura dinâmica. 20a ed. Concórdia: Santa Catarina; 1998

Recebido em: 24/03/2021

Aceito em: 29/07/2021 